\title{
Stochastic Analysis of Some Expedited Forwarding Networks
}

\author{
Milan Vojnović and Jean-Yves Le Boudec
}

\begin{abstract}
We consider stochastic guarantees for networks with aggregate scheduling, in particular, Expedited Forwarding (EF). Our approach is based on the assumption that a node can be abstracted by a service curve, and the input flows are regulated individually at the network ingress. Both of these assumptions are inline with the current definition of EF [1], [2]. We derive bounds to the complementary distributions of the backlog, delay through a single node, and the end-to-end delay. We also give a bound on the lossratio. Our analysis is exact under the given assumptions. Our results should help us to understand the performance of networks with aggregate scheduling, and provide the basis for dimensioning such networks.
\end{abstract}

Keywords - Expedited Forwarding, diffserv, Aggregate Scheduling, Statistical Multiplexing, Stochastic QoS, Service Curve, Packet Scale Rate Guarantee, Queueing, Loss Ratio

\section{INTRODUCTION}

$\mathrm{E}$ XPEDITED FORWARDING (EF) is a per-hop behavior (PHB) of the differentiated services (diffserv) Internet [1], [2]. With the EF PHB, individual flows (called "micro-flows", or "inputs" in this paper) are shaped separately at network access; from there on, they are served in an aggregate manner. Our objective is to derive probabilistic guarantees for EF network under the following assumptions.

Our approach is based on assumption that the EF PHB can be abstracted by a service curve.

(A1) We suppose a node offers to the aggregate of all EF traffic a service curve $\beta$, that is

$$
A^{*}(t) \geq A(s)+\beta(t-s), \text { for some } s \leq t,
$$

where $A^{*}(t)$ is the output data from the node on $[0, t]$ and $A(t)$ is the data which is accepted for service (i.e. not lost) at the input of the node during $[0, t][3],[4],[5]$.

The most recent definition of EF PHB (Def. IV.1 in [6] or DEF_1 in [1]), referred to as Packet Scale Rate Guarantee (PSRG), implies the service curve property, where $\beta$ has the form $\beta(t)=c(t-e)^{+}$(this is called a rate-latency service curve, with rate $c$ and latency $e$ ). A special case of PSRG is a scheduler that gives a static non-preemptive priority to EF traffic over non-EF traffic; here the rate is

The authors are with EPFL, Lausanne, Switzerland. E-mail: \{milan.vojnovic,jean-yves.leboudec\}@epfl.ch the server rate and the latency is the service time of the maximum-length non-EF packet. In Section IV, we use another property of PSRG, namely, the fact that delay can be bounded from backlog.

(A2) We suppose that the EF traffic inputs (micro-flows) at the network ingress points are mutually independent.

This assumption is also made in other work [7]. Note that we make no independence assumption for flows inside the network.

(A3) We suppose each EF input (micro-flow) at the network ingress point is regulated, that is to say, for a given input $i$ there exists a wide-sense increasing function $\alpha_{i}$ such that

$$
A_{i}^{0}(t)-A_{i}^{0}(s) \leq \alpha_{i}(t-s), \text { for any } s \leq t,
$$

where $A_{i}^{0}(t)$ is the data observed on $[0, t]$ of the input $i$ at the network ingress point.

In general, we derive our results for arbitrary arrival curves, and, in particular, we study the leaky-bucket regulated inputs; $\alpha_{i}(t)=\rho_{i} t+\sigma_{i}$.

(A4) We suppose $\mathbb{E}\left[A_{i}^{0}(t)-A_{i}^{0}(s)\right] \leq \rho_{i}(t-s)$, for any $s \leq t$, where

$$
\rho_{i}=\lim _{t \rightarrow \infty} \frac{\alpha_{i}(t)}{t} .
$$

Indeed, the assumption (A4) is implied for the input flows with stationary and ergodic increments [8], [9], but not vice versa. Thus, (A4) is a weaker assumption. Note that we allow for the input flows with non-stationary increments as long as (A4) is verified. However, for some of our results we need stationary ergodic increments of the inputs to ensure certain limits exist; we explicitly indicate when such an assumption is needed.

We now explain the organization of the paper and highlight our main findings. We discuss the state of the art in Section II. In Section III we give the theoretical foundations of our work; the results given in this section are of general interest for statistical multiplexing of regulated inputs to a multiplexer that offers a service curve to the aggregate input. Our prior work [9] gives us a catalog of probabilistic bounds to the backlog for the latter system. In Section III-A we go a step further and give a bound on the backlog that accommodates heterogeneously regulated inputs (Theorem 1), and which performs better than the 
bounds of Theorems 4 and 5 in [9]. Moreover, in Theorem 2, for a special case of leaky-bucket regulated inputs, we give three bounds to the complementary distribution of the backlog. A remarkable feature of the three bounds is that they are functions of some aggregate parameters of the leaky-bucket regulators.

In order to derive a probabilistic bound on the delay of a packet through a node we need an upper bound on the complementary distribution of the backlog as seen at packet arrival epochs. In Section III-B we find an inequality between the complementary distribution of the backlog seen at arrival epochs and the steady-state complementary distribution of the backlog (as seen at a randomly chosen point). In fact, we prove a more general result in Theorem 3 , and then specialize the result to the complementary distribution in Corollary 1.

When evaluating the performance of statistical multiplexing, a common performance metric studied is the probability that the buffer is above a given level. However, a performance metric of practical relevance is the loss ratio, which is a fraction of the data lost over a long time interval. In Theorem 4 (Section III-C) we give an exact upper bound on the loss rate and loss ratio in terms of the complementary distribution of the backlog process, a deterministic bound on the loss ratio [10], [11], and the aggregate arrival curve. We study the many small sources asymptotic regime in Section III-D; we identify the dominant probability and Bahadur-Rao asymptotic bounds of our exact bound in Theorem 1, and also discuss the typical time scale to overflow for leaky-bucket regulated inputs.

In Section IV we apply our findings to EF. We show how to obtain a probabilistic delay bound, based on the delay-from-backlog bounds of PSRG nodes. Then we apply a majorization by fresh traffic in order to find bounds at any node inside the network. Lastly, we explain different approaches, both exact and approximate, to compute an upper bound on the complementary distribution of the end-to-end delay through a sequence of nodes.

Section V studies our analytical results through numerical computations. We conclude the paper in Section VI. Proofs of the theorems are deferred to Appendix.

\section{RELATED WORK}

One approach to study EF is to derive deterministic bounds; this is pursued by Charny and Le Boudec in [12] and Bennett, Benson, Charny, Courtney, and Le Boudec in [6]. A worst-case bound on the delay jitter in [12], for leaky-bucket regulated EF input flows, is sup-linear in the maximum hop count, and it explodes at a certain utilization that may be rather low. Thus, the deterministic approach gives us hard QoS guarantees that may be quite pessimistic estimate of the performance. This leads us to seek for probabilistic guarantees.

An alternative probabilistic approach is proposed by Bonald, Proutière, and Roberts [7]. Their approach relies on two main assumptions. First, the EF traffic at the network ingress is better than Poisson meaning that the virtual waiting time distribution for the EF input traffic to a single node is stochastically smaller than if the input is replaced with a Poisson process with the same intensity as the original input. Second, it uses a conjecture that the jitter is negligible, which would ensure, if the EF traffic is better than Poisson at the network ingress, it remains so as the EF traffic passes through a sequence of nodes in the network. A remarkable property of the better than Poisson approach is that it is parsimonious in the parameters needed to characterize the input traffic - it requires only the intensity of the input. However, it is not clear whether the EF traffic would be better than Poisson, and whether the negligible jitter conjecture would be verified. Our approach does not make such assumptions, and our analysis is exact under our set of assumptions. In addition, [7] assumes that a node offers a static non-preemptive priority for EF traffic over non-EF traffic. Our results are valid for a node that offers a service curve, and thus accommodate the static priority system, but also other systems as discussed earlier.

In our prior work [9] we derive probabilistic bounds to the backlog for a network element that offers a service curve to the aggregate of independent regulated flows. The catalog of the bounds given there consists of two sets of the bounds. The first set of bounds is derived upon the virtual segregation of the backlog to individual input flows, and then observing that such virtual backlogs are with bounded support we applied Hoeffding's inequalities [13] to obtain closed-form bounds for both homogeneously and heterogeneously regulated inputs. It turns out that the bound for homogeneously regulated inputs generalizes the result of Kesidis and Konstantopoulos [14], which is for a workconserving constant service rate server. The second set of bounds is derived upon an approach originally due to Chang, Song, and Chiu [8] for a work-conserving constant service rate server. Our extension is to a super-additive service curve. Moreover, we derive bounds that hold exactly in continuous time and improve upon the bound in [8]. We also give two closed-form bounds for heterogeneously regulated inputs. In the present paper, we use the second set of bounds since it is shown that the bounds of the second set exhibit superior tightness than the bounds of the first set [9], [8]. 


\section{THEORETICAL FOUNDATIONS}

We introduce some more notation. For the input aggregate $A(t)$, consisting of $I$ flows, we write $A(t)=$ $\sum_{i=1}^{I} A_{i}(t)$. Also, the aggregate arrival curve is denoted as $\alpha(t)=\sum_{i=1}^{I} \alpha_{i}(t)$, and the upper bound on the aggregate sustainable rate as $\rho=\sum_{i=1}^{I} \rho_{i}$. Lastly, $\lambda_{\rho}(t):=$ $\rho t 1\{t \geq 0\}$.

In Lemma 1 [9], for $\beta$ super-additive, we show that $Q(t) \leq \tilde{Q}(t)$, for any $t$, where

$$
\tilde{Q}(t)=\sup _{t-\tau \leq s \leq t}\{A(t)-A(s)-\beta(t-s)\} .
$$

where $\tau=\inf \{u \geq 0 \mid \alpha(u) \leq \beta(u)\}$.

We say that $\beta$ is super-additive if $\beta(t+s) \geq \beta(t)+\beta(s)$, for all $t, s \geq 0$. Many service curves are super-additive, but not all. A sufficient condition is that $\beta$ is convex; in particular, the rate-latency service curve is super-additive, thus this additional assumption is not restrictive for our application to EF.

Note that $\tau$ is the intersection of the aggregate arrival curve $\alpha$ and the service curve $\beta$. Intuitively, think of $\tau$ as an upper bound on the busy period. This is formally correct if $\beta$ is a strict service curve $\beta$ (the service curve $\beta$ is strict if in addition to (1) the backlog $Q(s)=0$, for $s$ given in (1)).

For two functions $f, g$, define $v(f, g)=\sup _{u>0}\{f(u)-$ $g(u)\}$. For example, $v(\alpha, \beta)$ is the required buffer size to ensure loss-free operation.

We use $\tilde{Q}(\cdot)$ defined above to obtain an upper bound to the complementary distribution of $\tilde{Q}(\cdot)$, and thus an upper bound to the complementary distribution of $Q(\cdot)$.

\section{A. An Improved Bound on Backlog}

Theorem 1: Consider a node that offers a super-additive service curve $\beta$. Then, under (A1)-(A4), for any $t$, it holds

$$
\begin{aligned}
& \mathbb{P}(Q(t)>b) \leq \mathbb{P}(\tilde{Q}(t)>b) \leq \\
& \leq \sum_{k=0}^{K-1} \exp \left(-\frac{2\left(\left(b+\beta\left(s_{k}\right)-\rho s_{k+1}\right)^{+}\right)^{2}}{\left(\sum_{i=1}^{I} \alpha_{i}\left(s_{k+1}\right)^{2}\right) \wedge\left(4 \sum_{i=1}^{I} v\left(\alpha_{i}, \lambda_{\rho_{i}}\right)^{2}\right)}\right),
\end{aligned}
$$

for any $K \in \mathbb{N}$, and any $0=s_{0} \leq s_{1} \leq \cdots \leq s_{K}=\tau$.

Proof: Appendix A.

The bound (3) and our other bounds in [9] satisfy the economy of scale, a notion originally introduced by Botvich and Duffield [15]. It means that if we scale $b$ and $\beta$ as $O(I)$, then the probability to overflow decays exponentially with $I$. We also note that with fixed aggregate arrival curve, the bound in (3) is tightest for all the inputs having identical arrival curves $\alpha_{i}(t)=\alpha(t) / I$. We call this the economy of equality; it tells us that we get the best performance if the input flows all have the same arrival curve constraint.
Next we give three bounds to the probability to overflow for leaky-bucket regulated inputs. The bounds require some aggregate knowledge about the leaky-buckets. As such, they merit is when the knowledge about the input aggregate to a node is limited, or if the per-flow state is not allowed, which is inline with diffserv philosophy.

Theorem 2: (Three Bounds to the Backlog for LeakyBucket Regulated Inputs) Consider a node that offers a super-additive service curve $\beta$, fed with leaky-bucket regulated inputs; $\alpha_{i}(t)=\rho_{i} t+\sigma_{i}$. Then, under (A1)-(A4), for any $t$, it holds

$$
\begin{aligned}
& \mathbb{P}(Q(t)>b) \leq \mathbb{P}(\tilde{Q}(t)>b) \leq \\
& \stackrel{(a)}{\leq} \sum_{k=0}^{K-1} \exp \left(-\frac{2\left(\left(b+\beta\left(s_{k}\right)-\rho s_{k+1}\right)^{+}\right)^{2}}{\left(\sum_{i=1}^{I}\left(\rho_{i} s_{k+1}+\sigma_{i}\right)^{2}\right) \wedge\left(4 \sum_{i=1}^{I} \sigma_{i}^{2}\right)}\right) \\
& \stackrel{(b)}{\leq} \sum_{k=0}^{K-1} \exp \left(-\frac{2\left(\left(b+\beta\left(s_{k}\right)-\rho s_{k+1}\right)^{+}\right)^{2}}{\left(\sqrt{\sum_{i=1}^{I} \rho_{i}^{2}} s_{k+1}+\sqrt{\sum_{i=1}^{I} \sigma_{i}^{2}}\right)^{2} \wedge\left(4 \sum_{i=1}^{I} \sigma_{i}^{2}\right)}\right) \\
& \stackrel{(c)}{\leq} \sum_{k=0}^{K-1} \exp \left(-\frac{2\left(\left(b+\beta\left(s_{k}\right)-\rho s_{k+1}\right)^{+}\right)^{2}}{\left(\rho s_{k+1}+\sqrt{\sum_{i=1}^{I} \sigma_{i}^{2}}\right)^{2} \wedge\left(4 \sum_{i=1}^{I} \sigma_{i}^{2}\right)}\right),
\end{aligned}
$$

for any $K \in \mathbb{N}$, and any $0=s_{0} \leq s_{1} \leq \cdots \leq s_{K}=\tau$.

Proof: Appendix B.

Let $\vec{\rho}=\left[\rho_{1}, \ldots, \rho_{I}\right]$ and $\vec{\sigma}=\left[\sigma_{1}, \ldots, \sigma_{I}\right]$ be the vectors of the sustainable rates and burstiness parameters, respectively. Consider the following aggregate parameters:

(P1) $\sum_{i=1}^{I} \rho_{i}-$ the aggregate sustainable rate,

(P2) $\sum_{i=1}^{I} \sigma_{i}^{2} \sim$ the second moment of $\vec{\sigma}$,

(P3) $\sum_{i=1}^{I} \rho_{i}^{2} \sim$ the second moment of $\vec{\rho}$,

(P4) $\sum_{i=1}^{I} \rho_{i} \sigma_{i} \sim$ the correlation of $\vec{\rho}$ and $\vec{\sigma}$.

Then, it is readily seen that the bounds in (4) require that we know upper bounds to: (a) (P1)-(P4), (b) (P1)-(P3), and (c) (P1)-(P2). It is a remarkable property that the bound (c) in (4) needs only two aggregate parameters, namely, (P1) and (P2). An issue of the interest is how much we loose in terms of tightness as we know fewer aggregate parameters. We explore this numerically in Section V.

\section{B. Bound on the Backlog at Arrival Epochs}

In the previous section we consider the steady-state complementary distribution of the backlog. This may be empirically interpreted as a fraction of time the backlog is above a given level (time average). Here we consider the complementary distribution of the backlog as seen by the packet arrivals, which may be empirically interpreted as a fraction of the arrival data that encounter the backlog above a given level (Palm average). We denote this as $\mathbb{P}_{A}$ for the arrival process $A$ ( $\mathbb{E}_{A}$ is the expectation with respect to $\mathbb{P}_{A}$ ).

Theorem 3: Consider a node that offers a service curve $\beta$. Define $\hat{\beta}=\sup _{t, u \geq 0} \frac{\beta(t+u)-\beta(t)}{u}$. Suppose the input $A$ is with stationary increments and intensity $\bar{\rho}<\hat{\beta}$. Then, 
for any measurable function $\psi: \mathbb{R}_{+} \rightarrow \mathbb{R}_{+}$,

$$
\mathbb{E}_{A}[\psi(\tilde{Q}(0))] \leq \frac{\hat{\beta}}{\bar{\rho}} \mathbb{E}[\psi(\tilde{Q}(0))]
$$

Proof: Appendix C.

Take $\psi(x)=1_{(b, \infty)}(x)$ in (5), then we directly obtain the following corollary.

Corollary 1: Under the assumptions of the theorem, it holds

$$
\mathbb{P}_{A}(\tilde{Q}(0)>b) \leq \frac{\hat{\beta}}{\bar{\rho}} \mathbb{P}(\tilde{Q}(0)>b) .
$$

Remark 1: Konstantopoulos and Last [16] study a work-conserving constant service rate server. They prove equality in (5) for any measurable function $\psi: \mathbb{R}_{+} \rightarrow \mathbb{R}$.

Remark 2: The corollary tells us that for the majorizing process $\tilde{Q}$, the complementary distribution of the backlog at arrival epochs is less than or equal to the steady-state complementary distribution of the backlog, times the ratio of the maximum slope of $\beta$ on $[0, \tau]$ and the intensity of the arrival process $\bar{\rho}$. For the rate-latency service curve $\beta(s)=$ $c(s-e)^{+}$, it reads as $\mathbb{P}_{A}(\tilde{Q}(0)>b) \leq \frac{c}{\bar{\rho}} \mathbb{P}(\tilde{Q}(0)>b)$.

Note that the result in Corollary 1 is established for a majorizing process $\tilde{Q}(\cdot)$ to the backlog $Q(\cdot)$. As such, it enables us to state:

$$
\mathbb{P}_{A}(Q(0)>b) \leq \frac{\hat{\beta}}{\bar{\rho}} \mathbb{P}(\tilde{Q}(0)>b) .
$$

\section{Bound on the Loss Ratio}

Insofar, we consider upper bounds to the complementary distribution of the backlog process. However, in practice, the real performance metric of the interest is the loss ratio (a fraction of data lost over a long time interval). Formally, let $L(t)$ be the data lost in $[0, t]$, then the loss ratio is defined as $\bar{l}=\lim _{t \rightarrow \infty} L(t) / A(t)$. The next theorem gives us an exact bound on the loss ratio in terms of the complementary distribution of the backlog. Thus, having identified an upper bound on the complementary distribution of the backlog, the next theorem enables us to directly obtain an upper bound on the loss ratio.

Theorem 4: Consider a node that offers a service curve $\beta$ and finite buffer capacity $B$. Let $Q^{*}(\cdot)$ be the backlog of a virtual system identical to the original system, but with a buffer size sufficient to ensure no losses. Let $\hat{l}(t)=1-$ $\inf _{s \leq t} \frac{\beta(s)+B}{\alpha(s)}$. Then, an upper bound on the loss rate is

$$
\begin{aligned}
\mathbb{E}[L(t)-L(t-1)] & \leq \int_{B}^{B+\hat{l}(1) \alpha(1)} \mathbb{P}\left(Q^{*}(t)>z\right) d z \\
& \leq \hat{l}(1) \alpha(1) \mathbb{P}\left(Q^{*}(t)>B\right)
\end{aligned}
$$

Moreover, for ergodic inputs with stationary increments, and the intensity of the aggregate input $\bar{\rho}$, an upper bound on the loss ratio is

$$
\begin{aligned}
\bar{l} & \leq \frac{1}{\bar{\rho}} \int_{B}^{B+\hat{l}(1) \alpha(1)} \mathbb{P}\left(Q^{*}(0)>z\right) d z \\
& \leq \frac{\hat{l}(1) \alpha(1)}{\bar{\rho}} \mathbb{P}\left(Q^{*}(0)>B\right) .
\end{aligned}
$$

Proof: Appendix D.

Remark 3: A similar expression to (6) was obtained by Likhanov and Mazumdar [17] for a work-conserving constant service rate system in the regime of the many sources asymptotics. Our result shows us that the bounds on the loss rate and loss ratio hold exactly (not only asymptotically). Moreover, we show it holds for a service curve network element, which encompasses a work-conserving constant service rate system, and thus it is more general. Lastly, for regulated input flows, we improve the bound on the loss rate by tightening the upper boundary of the integral in (6) from $+\infty$ to $B+\hat{l}(1) \alpha(1)$. It can be readily shown that $\hat{l}(t) \alpha(t) \leq v(\alpha, \beta)-B$, for all $t \geq 0$, and thus the bounds in (6) and (7) are better than if we use $v(\alpha, \beta)-B$ in place of $\hat{l}(1) \alpha(1)$.

Example 1: Consider leaky-bucket regulated inputs $\alpha_{i}(t)=\rho_{i} t+\sigma_{i}$ with $\sum_{i=1}^{I} \rho_{i}=\rho$ and $\sum_{i=1}^{I} \sigma_{i}=\sigma$. Suppose the rate-latency service curve $\beta(t)=c(t-e)^{+}$. Then,

$$
\hat{l}(t)= \begin{cases}1-\frac{B}{\rho t+\sigma}, & t \leq e \\ 1-\frac{B}{v(\alpha, \beta)}, & t>e,\end{cases}
$$

where $v(\alpha, \beta)=\rho e+\sigma$.

Indeed, $\hat{l}(t)$ is a hard deterministic bound to the loss ratio over any interval of length $t$ [11], [10]. It is evident that (8) is overly conservative with respect to (7).

\section{Many Small Sources Asymptotics}

In the preceding sections and [9], the bounds we derived are exact. The bounds hold exactly for any setting of the parameters, and in particular, the bounds are valid for any number of the input flows. In this section we consider asymptotic counterparts to the bounds given earlier. In particular, we study the many small sources asymptotics - the buffer size and capacity scale as $O(I)$, where the number of the input flows $I$ tends to infinity.

We will see that in the asymptotic regime our bounds admit a simpler form. One reason to study the asymptotic results is to gain insight how the bounds behave, in particular, what is the most likely way the backlog build up.

We have

$$
\begin{aligned}
& \mathbb{P}(Q(0)>b) \leq \sum_{k=0}^{K-1} \mathbb{P}\left(A(0)-A\left(-s_{k+1}\right)>b+\beta\left(s_{k}\right)\right) \\
& \leq \sum_{k=0}^{K-1} \mathbb{P}\left(A(0)-A\left(-s_{k}\right)>b+\beta\left(s_{k}\right)-\alpha\left(s_{k+1}-s_{k}\right)\right) \\
& \leq \sum_{k=0}^{K-1} \mathbb{P}\left(A(0)-A\left(-s_{k}\right)>b+\beta\left(s_{k}\right)-\alpha(\delta)\right),
\end{aligned}
$$


for any $K \in \mathbb{N}$, and any $0=s_{0} \leq s_{1} \leq \cdots \leq s_{K}=\tau$, where $\delta=\max _{k \in\{0,1, \ldots, K-1\}}\left\{s_{k+1}-s_{k}\right\}$. We note that the first inequality is from [9], and the second and third inequalities are evident by definition of $\alpha$.

Suppose the $k$-th summation term in the last inequality of (9) is bounded with $e^{-g\left(s_{k}\right)}$, for some positive-valued function $g$. Suppose $0=s_{0} \leq s_{1} \leq \cdots \leq s_{K}=\tau$ is such that there exists a unique $k^{*} \in\{0,1, \ldots, K-1\}$ and $\epsilon>0$ such that $g\left(s_{k^{*}}\right)+I \epsilon \leq g\left(s_{k}\right)$, for all $k \in$ $\left\{0,1, \ldots, K-1 \mid k \neq k^{*}\right\}$.

Then, for the many small sources scaling $g(\cdot) \sim O(I)$, and

$$
\mathbb{P}(Q(0)>b) \leq e^{-g\left(s^{*}\right)}\left(1+O\left(e^{-I \epsilon}\right)\right),
$$

as $I \rightarrow \infty$, where $s^{*} \in[0, \tau]$ such that $g\left(s^{*}\right)=$ $\inf _{s \in[0, \tau]} g(s)$. Note that (10) does not require $s^{*}$ to be unique on $[0, \tau]$; however, the partition $0=s_{0} \leq s_{1} \leq$ $\cdots \leq s_{K}=\tau$ need to ensure a unique minimum of $\left\{g\left(s_{0}\right), g\left(s_{1}\right), \ldots, g\left(s_{K-1}\right)\right\}$. If $s^{*}$ is unique on $[0, \tau]$, then it may be interpreted as the typical time scale (e.g. [17], [8]) to overflow a given level of the buffer.

We readily identify the function $g$ for our bound in (3)

$$
g(s)=\frac{2\left((b+\beta(s)-\rho s-\alpha(\delta))^{+}\right)^{2}}{\left(\sum_{i=1}^{I} \alpha_{i}(s)^{2}\right) \wedge\left(4 \sum_{i=1}^{I} v\left(\alpha_{i}, \lambda_{\rho_{i}}\right)^{2}\right)} .
$$

Likewise, one may derive the many small sources asymptotics for our other bounds in [9].

Note that (10) holds for any $K \in \mathbb{N}$. We can take a uniform partition of $[0, \tau]$ such that $\delta=\tau / K$, and then let $K \rightarrow \infty$. This allows us to replace $\alpha(\delta)$ in (11) with $\lim _{\delta \downarrow 0} \alpha(\delta)$. For a right-hand continuous $\alpha$ at 0 , we replace $\alpha(\delta)$ with $\alpha(0)$; if, in addition, $\alpha(0)=0$, the term $\alpha(\delta)$ in (11) vanishes. The term indeed vanishes for peakrate constrained aggregate input. In the further notation we keep $\alpha(\delta)$, but the above observations hold.

In the particular case of a node that offers a rate-latency service curve, fed with leaky-bucket regulated inputs, we can show that $s^{*}$ is unique, and it is equal to

$$
s^{*}=\left(u \wedge \tau_{c} \wedge \tau\right) \vee e,
$$

where

$$
u=\frac{\frac{b-c e-\alpha(\delta)}{c-\rho} \sum_{i=1}^{I} \rho_{i} \sigma_{i}-\sum_{i=1}^{I} \sigma_{i}^{2}}{\sum_{i=1}^{I} \rho_{i} \sigma_{i}-\frac{b-c e-\alpha(\delta)}{c-\rho} \sum_{i=1}^{I} \rho_{i}^{2}},
$$

for $b<b^{*}$, otherwise, set $u=\tau$, for $b \geq b^{*}$, and

$$
\tau_{c}=\frac{\sum_{i=1}^{I} \rho_{i} \sigma_{i}}{\sum_{i=1}^{I} \rho_{i}^{2}}\left(\sqrt{1+3 \frac{\sum_{i=1}^{I} \rho_{i}^{2} \sum_{i=1}^{I} \sigma_{i}^{2}}{\left(\sum_{i=1}^{I} \rho_{i} \sigma_{i}\right)^{2}}}-1\right),
$$

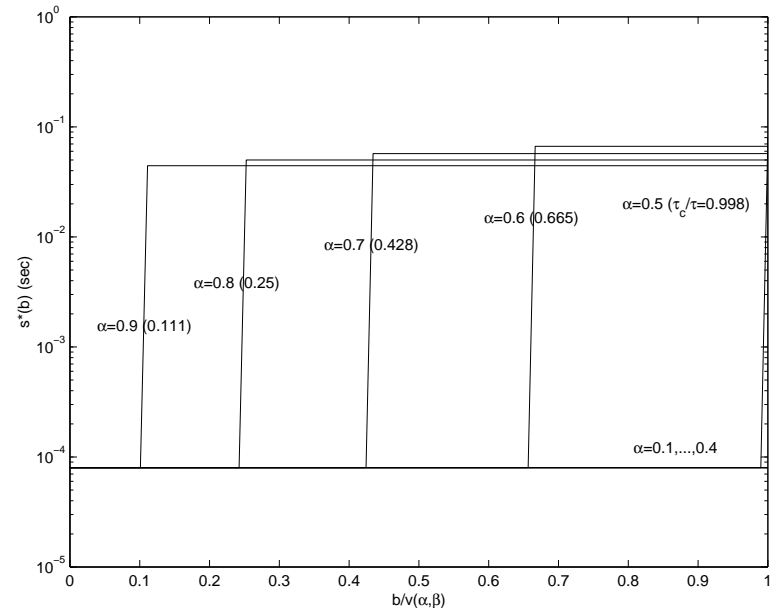

Fig. 1. The typical time-scale to overflow versus the buffer size $b$. The setup us is as follows: the inputs are homogeneous, $I=100$, MTU $=1500$ Bytes, $c=150 \mathrm{Mbps}, e=\mathrm{MTU} / c$, and $\sigma_{1}=5 \mathrm{MTU}$. The number in the brackets denotes $\tau_{c} \wedge \tau$ as a fraction of $\tau$.

and

$$
b^{*}=(c-\rho) \frac{\sum_{i=1}^{I} \rho_{i} \sigma_{i}}{\sum_{i=1}^{I} \rho_{i}^{2}}+c e+\alpha(\delta) .
$$

We note that $\tau_{c}$ is a value of $s$ in (11) at which, in the minimum operation, the first term becomes larger than the second term. On the other hand, $b^{*}$ is a cut-off buffer size at which the typical time scale to overflow turns from one value to another. In Fig. 1 we show numerical values for $s^{*}$ versus the buffer size $b$, for one particular example. For the utilization larger than 0.5 , we observe that $s^{*}$ is equal to $e$ for $b<b^{*}$ and then it is equal to $\tau_{c} \wedge \tau$ for $b \geq b^{*}$.

We compare with the bounds of Theorems 4 and 5 in [9] for leaky-bucket regulated inputs:
(B1) $\sum_{k=0}^{K-1} \exp \left(-\frac{2\left(\left(b+\beta\left(s_{k}\right)-\rho s_{k+1}\right)^{+}\right)^{2}}{\sum_{i=1}^{I}\left(\rho_{i} s_{k+1}+\sigma_{i}\right)^{2}}\right)$
(B2) $\sum_{k=0}^{K-1} \exp \left(-\frac{\left(\left(b+\beta\left(s_{k}\right)-\rho s_{k+1}\right)^{+}\right)^{2}}{2 \sum_{i=1}^{I} \sigma_{i}^{2}}\right)$.

Similar but different bounds than (B1) and (B2) are obtained by majorization (9), which amounts to replacing $s_{k+1}$ with $s_{k}$ and adding $-\alpha(\delta)$ term within $(\cdot)^{+}$. It can be shown that for the former bound, $s^{*}=e$ for $b<b^{*}$, and $s^{*}=\tau$ for $b \geq b^{*}$. For the latter bound, we have $s^{*}=e$. Thus, with the former bound, for the buffer sizes larger than $b^{*}$, the typical time scale to overflow becomes $\tau$, which may be quite large, in particular, for high utilization. Our bound (3) lessen the latter effect since the typical time scale to overflow for $b \geq b^{*}$ is reduced to $\tau_{c} \wedge \tau$.

We readily obtain Bahadur-Rao [18] bound, as $I \rightarrow \infty$,

$$
\mathbb{P}(Q(0)>b) \approx \frac{1}{\sqrt{4 \pi \Lambda\left(s^{*}\right)}} e^{-\Lambda\left(s^{*}\right)},
$$


where

$$
\Lambda\left(s^{*}\right)=-\frac{2\left(\left(b+\beta\left(s^{*}\right)-\rho s^{*}-\alpha(\delta)\right)^{+}\right)^{2}}{\sum_{i=1}^{I} \alpha_{i}\left(s^{*}\right)^{2}} .
$$

For identically distributed inputs (in our case, for the homogeneously regulated inputs) Likhanov and Mazumdar [17] (Proposition 2.1) show that (13) is an exact asymptotics up to a multiplicative constant $1+O(1 / I)$. Their result is under two assumptions: (1) $s^{*}$ is unique, and (2) $\liminf \operatorname{in}_{t \rightarrow \infty} \Lambda(t) / \ln t>0$. It can be shown that in our case (1) holds, and (2) is not needed given that we have a finite summation in (9). On the other hand, for heterogeneously regulated inputs, one may use the central limit approximation as discussed in [19] (Section 5.4). Indeed, the pre-factor in (13) scales as $1 / \sqrt{I}$, which was observed also elsewhere, e.g. by Montgomery and de Veciana [20]. We come back to the Bahadur-Rao bound in Section V, where we give a numerical example to demonstrate how much we improve with the Bahadur-Rao pre-factor.

\section{ApPLICATION TO EF}

\section{A. Delay-from-Backlog for a PSRG Node}

In general, for an arbitrary node, we cannot directly deduce a bound on the complementary distribution of the delay from the complementary distribution of the backlog. However, this is possible if the node can be abstracted with PSRG, as is the case for the proposed definition of EF. Indeed, a delay-from-backlog bound for PSRG nodes is given in [6] for FIFO nodes, and it is proven in [21] that it holds without the FIFO assumption.

Proposition 1: For a PSRG node with rate $c$ and latency $e$, it holds

$$
\mathbb{P}(d(0)>u) \leq \mathbb{P}_{A}(Q(0)>c(u-e)), \text { for } u \geq e,
$$

where $d(0)$ is a delay incurred by an arbitrary packet that arrives at time 0 .

Proof: By Theorem 1 in [6] and Theorem III.1 in [21], the delay for a packet arriving at time $t$ is bounded by $Q(t) / c+e$; then simply use this point-wise majorization to obtain (14).

Thus, combining (14) with Corollary 1 and any upper bound on the steady-state complementary distribution to the backlog (e.g., (3) or (4)), we obtain an upper bound on the complementary distribution of the delay.

\section{B. Majorization by the fresh traffic}

Our bounds in [9] and (3), and typically the bounds found elsewhere, are based on the assumption that the input flows are mutually independent. Thus, we cannot apply the bounds directly, because it is not realistic to sup- pose the input flows to any node in the network are mutually independent; the flows may get correlated as they share common upstream nodes. However, it is reasonable to suppose that the input flows at the network ingress are mutually independent (our assumption (A2) in Section I). We suppose that the delay jitter incurred at the upstream nodes to a given node is bounded by $\Delta$. Such a bound indeed exists with finite buffer sizes; use the delay-frombacklog [6], [21] to obtain $\Delta=(h-1) \max \left\{B_{n} / c_{n}+e_{n}\right\}$, where $h$ is the maximum hop count, $B_{n}$ is the buffer size, $c_{n}$ the service rate, and $e_{n}$ the latency of the node $n$. Then, we majorize increments of the input flows to a given node by the fresh traffic at the network ingress

$$
A_{i}(t)-A_{i}(s) \leq A_{i}^{0}(t)-A_{i}^{0}(s-\Delta) .
$$

Originally, such a majorization is suggested by Chang, Chiu, and Song [8]. In particular, for our bounds in (3) and (4) this amounts to replace $s_{k+1}$ with $s_{k+1}+\Delta$. We discuss in the next section how we can obtain a tighter bound on the delay jitter incurred at the upstream nodes to a given node in computing a probabilistic bound on the end-to-end delay. It is our work in progress to investigate whether our bounds can be applied directly for some non-independent input flows. We note that we can easily generalize our bounds to non-independent input flows by using Hölder's inequality in our application of ChernoffHoeffding's bounds. Then, it can be shown that all our bounds to the backlog remain the same, but with the exponent divided with $I$; this precludes the statistical multiplexing gain.

\section{Delay Through a Sequence of Nodes}

Let $d_{n}$ be the delay of an arbitrary packet through the node $n$. Suppose the packet traverses $h$ nodes. Thus, the end-to-end delay incurred by the packet is

$$
d=d_{1}+d_{2}+\cdots+d_{h}
$$

In Section IV-A we show how to derive an upper bound $F_{n}(u)$ to $\mathbb{P}\left(d_{n}>u\right)$. The next issue is to obtain an upper bound on $\mathbb{P}(d>u)$, which we denote as $G(u)$.

Our first approach gives us $G(u)$ that holds exactly,

$$
G(u)=\sum_{n=1}^{h} F_{n}\left(\frac{u}{h}\right) .
$$

This is readily shown by noting $d \leq h \max \left\{d_{1}, \ldots, d_{h}\right\}$, and then

$$
\begin{aligned}
\mathbb{P}(d>u) & \leq \mathbb{P}\left(\max \left\{d_{1}, \ldots, d_{h}\right\}>\frac{h}{u}\right) \\
& \leq \mathbb{P}\left(\bigcup_{n \in\{1, \ldots, h\}}\left\{d_{n}>\frac{u}{h}\right\}\right) \\
& \leq \sum_{n=1}^{h} \mathbb{P}\left(d_{n}>\frac{u}{h}\right) \leq \sum_{n=1}^{h} F_{n}\left(\frac{u}{h}\right) .
\end{aligned}
$$


As (16) is derived by summing up $h$ times the maximum delay along the route, it may yield a conservative bound on the end-to-end delay. Clearly, (16) is sup-linear in the hop count $h$.

Our second approach holds for associated $\left\{d_{1}, \ldots, d_{h}\right\}$. The sequence $\left\{d_{1}, \ldots, d_{h}\right\}$ is associated ([22], Sec. 4.3.1) if, in particular, for all non-decreasing mappings $f_{n}$ : $\mathbb{R}^{h} \rightarrow \mathbb{R}, \mathbb{E}\left[\prod_{n=1}^{h} f_{n}\left(d_{n}\right)\right] \geq \prod_{n=1}^{h} \mathbb{E}\left[f_{n}\left(d_{n}\right)\right]$. Obviously, for mutually independent $d_{1}, \ldots, d_{h}$, the sequence $\left\{d_{1}, \ldots, d_{h}\right\}$ is associated. The sequence $\left\{\bar{d}_{1}, \ldots, \bar{d}_{h}\right\}$ is said to be an independent version of $\left\{d_{1}, \ldots, d_{h}\right\}$ if $\bar{d}_{1}, \ldots, \bar{d}_{h}$ are mutually independent, and $d_{n}$ and $\bar{d}_{n}$ have the same distribution for all $n=1, \ldots, h$. It can be shown $\mathbb{P}\left(\max \left\{d_{1}, \ldots, d_{h}\right\}>u\right) \leq \mathbb{P}\left(\max \left\{\bar{d}_{1}, \ldots, \bar{d}_{h}\right\}>u\right)$, which gives us a product-form bound

$$
G(u)=1-\prod_{n=1}^{h}\left(1-F_{n}\left(\frac{u}{h}\right)\right) .
$$

Indeed,

$$
\begin{aligned}
& \mathbb{P}\left(\max \left\{\bar{d}_{1}, \ldots, \bar{d}_{h}\right\}>\frac{u}{h}\right)=1-\mathbb{P}\left(\max \left\{\bar{d}_{1}, \ldots, \bar{d}_{h}\right\} \leq \frac{u}{h}\right) \\
& =1-\mathbb{P}\left(\bigcap_{n \in\{1, \ldots, h\}}\left\{\bar{d}_{n} \leq \frac{u}{h}\right\}\right) \\
& =1-\prod_{n=1}^{h} \mathbb{P}\left(\bar{d}_{n} \leq \frac{u}{h}\right) .
\end{aligned}
$$

Note in $(17), G(u) \approx \sum_{n=1}^{h} F_{n}\left(\frac{u}{h}\right)$, with $F_{n}\left(\frac{u}{h}\right) \ll 1$ for all $n=1, \ldots, h$. Thus, in this case, we do not expect (17) to be substantially superior than (16).

Our third approach is an approximation that may give a tighter bound on the end-to-end delay. It is based on assuming the delays incurred at different hops are independent. Such an assumption is also made in [7]. Then, by (15), the end-to-end delay is a $h$-fold convolution of $F_{1}(u)$ to $F_{h}(u)$.

As discussed in Section IV-B, for the majorization by the fresh traffic, $F_{n}(u)$ is also function of $\Delta$, a bound on the delay jitter incurred at the upstream nodes to the node $n$. From the delay-from-backlog bound [6], [21], we know that we can use $\Delta=(h-1) \max _{n \in\{1, \ldots, h\}}\left\{\frac{B_{n}}{c_{n}}+e_{n}\right\}$, where $B_{n}$ is the buffer size, $c_{n}$ the service rate, and $e_{n}$ the latency at the node $n$. An improvement may be achieved by a further approximation. Let $G(u, \Delta)$ be $G(u)$ in (16), with the upper bound on the upstream delay jitter equal to $\Delta$. Then, given there is a unique $\Delta_{\epsilon} \geq 0$ such that $G\left(\Delta_{\epsilon}, \Delta_{\epsilon}\right)=\epsilon$, for some small $0<\epsilon<1$, we obtain an upper bound on the delay jitter at the upstream nodes that does not hold with probability 1 , but with a high probability.

The latter approach uses a uniform bound on the delay jitter incurred at the upstream nodes to any node in the network. An alternative approach goes as follows. We again suppose the delays incurred at different nodes are independent. But, we take as an upper bound on the delay jitter incurred at the upstream nodes to the node $n$ as $\Delta$, given that $d_{1}+\cdots+d_{n-1}=\Delta$. This effectively amounts to that $d_{1}, \ldots, d_{h}$ are made dependent due to our majorization by the fresh traffic. This gives us the following recursion for the upper bound on $\mathbb{P}\left(d_{1}+\cdots+d_{n}>u\right)$, which we denote as $G_{n}(u)$,

$$
G_{n}(u)=\int_{0}^{u} F_{n}(u-y, y) d G_{n-1}^{c}(y)+G_{n-1}(u),
$$

where $G_{n}^{c}(\cdot)=1-G_{n}(\cdot)$, and $\mathbb{P}\left(d_{n}>u-y \mid d_{1}+\cdots+\right.$ $\left.d_{n-1}=y\right) \leq F_{n}(u-y, y)$.

Lastly, if one prefers simplicity over tightness, for logconcave $F_{n}(u)$, for all $n=1, \ldots, n$, one may use an exponential upper bound on $F_{n}(u), n=1, \ldots, n$, and then $G(u)$ is simply $h$-Erlang complementary distribution.

A detailed evaluation of the tightness of bounds to the complementary distribution of the end-to-end delay presented in this section is left for further study.

\section{NumericAl Results}

We compare numerically the bounds given in (4). We consider two traffic classes, each consisting of $I_{j}=50$ flows, $j=1,2$. Class- $j$ flows are $\left(\rho_{j}, \sigma_{j}\right)$ leaky-bucket regulated. The service curve is the rate-latency with $c=$ $150 \mathrm{Mbps}$ and $e=\mathrm{MTU} / c$, where MTU=1500 Bytes. We also compare with the bounds (B1) and (B2) given in Section III-D. The bounds are computed as the infimum over uniform partition of $[0, \tau]$, thus $s_{k}=k \tau / K$, for $k=0, \ldots, K$.

In Fig. 2 (two upper graphs), we show the bounds for the homogeneous case ( $\vec{\rho}$ and $\vec{\sigma}$ non-correlated); in Fig. 2 (two lower graphs) we show the bounds for the heterogeneous case ( $\vec{\rho}$ and $\vec{\sigma}$ positively correlated). We make the following observations. First, (B1) is better than (B2) in most of the cases, except for high utilization. The bound (a) in (4) is very close to the bound (B1) for low to moderate utilization. For high utilization, (a) in (4) fixes the deviation of (B1). Second, bounds (b) and (a) in (4) are close to each other in all the cases. Third, bound (c) in (4) is fairly close to the bound (a) in (4) for low utilization. As the utilization increases, (c) in (4) moves gradually from (B1) to (B2). Forth, the deviation of (B2) for high utilization is indeed stronger for positively correlated $\vec{\rho}$ and $\vec{\sigma}$ and larger $\sum_{i=1}^{I} \sigma_{i}^{2}$. Last, but not least, (B1) and (a)-(b) in (4) are insensitive to the utilization (i.e. $\vec{\rho}$ ) for low to moderate utilization.

Our earlier numerical results indicate that with the bounds (B1), (B2), and (a)-(b) in (4) we have insensitivity 

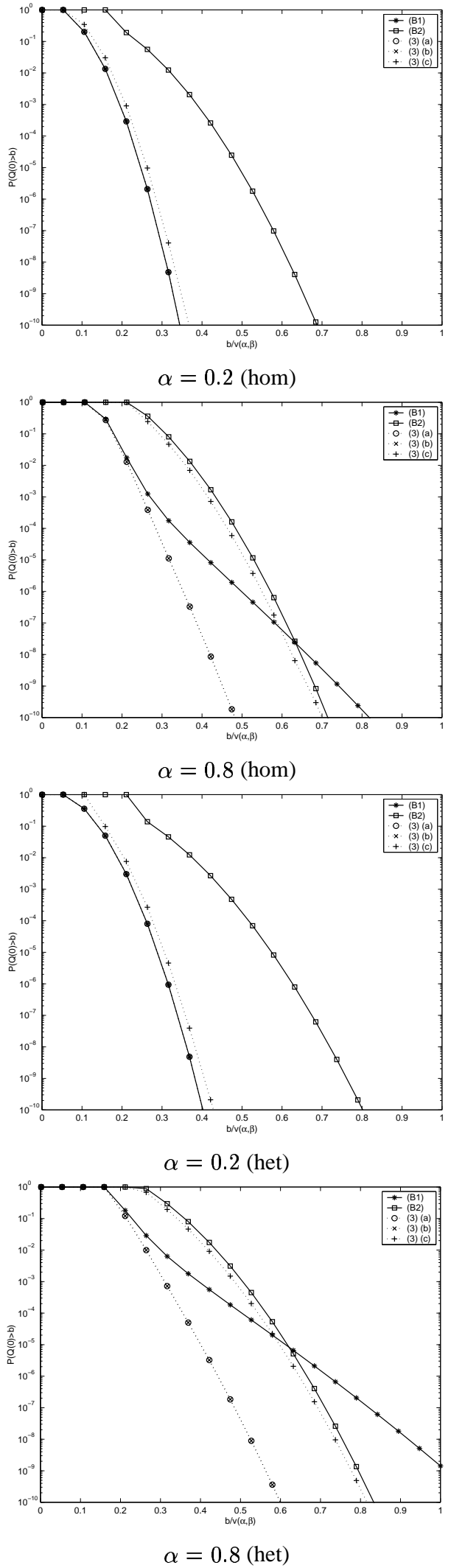

Fig. 2. Bounds with input aggregate of two traffic classes. Class- $j$ consists of $I / 2\left(\rho_{j}, \sigma_{j}\right)$ leaky-bucket regulated inputs. The two upper graphs are for homogeneously regulated inputs $\rho_{j}=\rho / I, \sigma_{j}=5 \mathrm{MTU}$. The two lower graphs are for heterogeneously regulated inputs $\rho_{1}=0.2 \alpha c /(I / 2)$, $\rho_{2}=0.8 \alpha c /(I / 2), \sigma_{1}=2 \mathrm{MTU}, \sigma_{2}=8 \mathrm{MTU}$. Other parameters are set as $c=150 \mathrm{Mbps}$, MTU $=1500$ Bytes, $e=\mathrm{MTU} / c$.
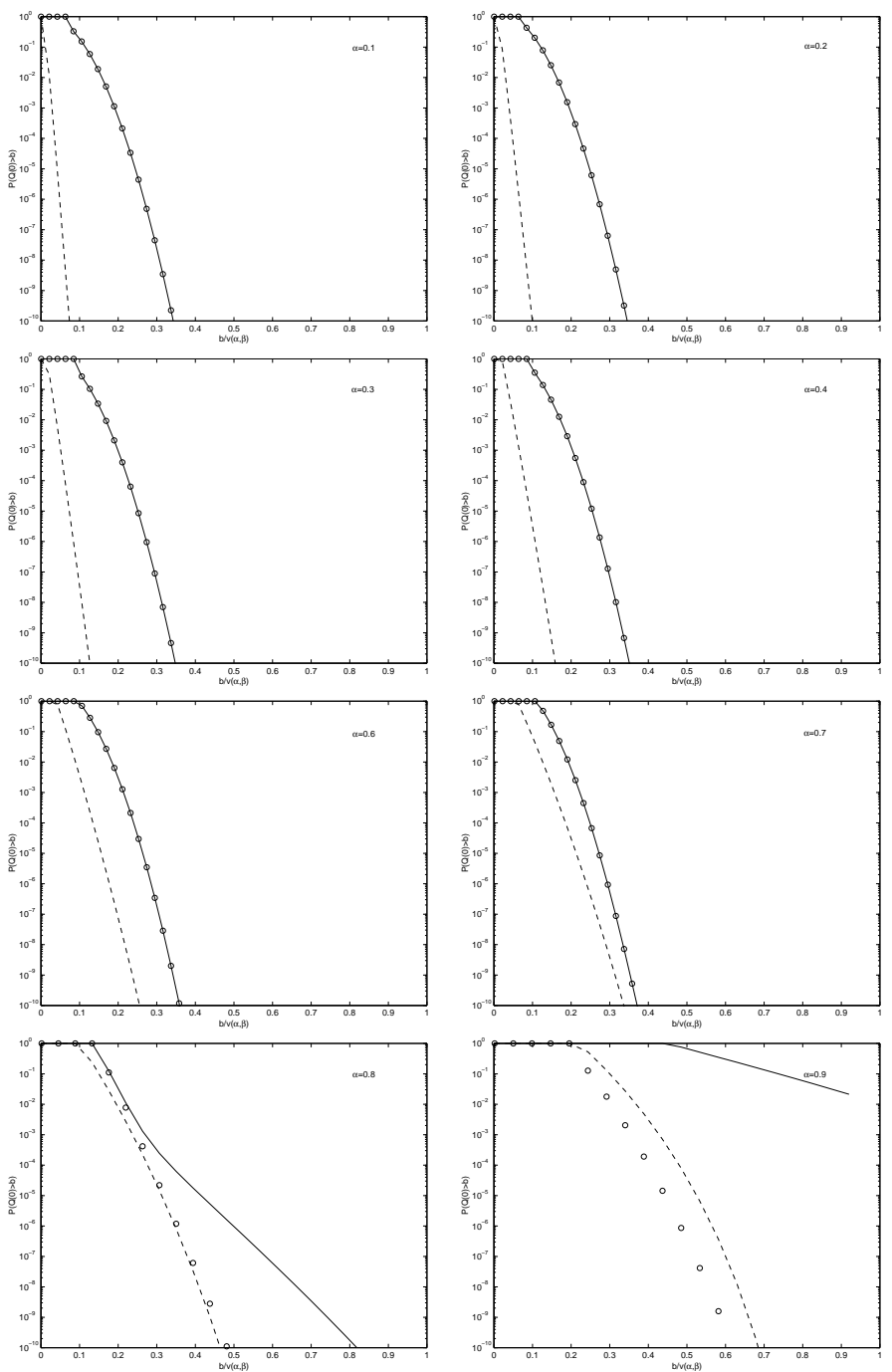

Fig. 3. Bound (a) in (4) and (B1) versus the homogeneous counterpart to (B1) (Theorem 3 in [6]). The circles show the bound (a) in (4); the solid line is for (B1); the dashed line is for the homogeneous counterpart to (B1).

with respect to $\vec{\rho}$ for low to moderate utilization. Asymptotic analysis tells us that the typical time scale to overflow is a small fraction of $\tau$ in those cases, and thus, in the denominator of the exponents in (B1), (B2), and (a)-(b) in (4), a major impact is attributed to the burstiness parameters, i.e. $\sum_{i=1}^{I} \sigma_{i}^{2}$.

Next, we confront our bound (B1) to its counterpart derived under the assumption that the input flows are homogeneously regulated (Theorem 3 in [9]). In Fig. 3, we show the bounds (B1), (a) in (4), and the homogeneous counterpart to (B1) versus the normalized buffer size. We observe that for low utilization (B1) and (a) in (4) are conservative with respect to the homogeneous counterpart of (B1). However, for high utilization the discrepancy between the bound (a) in (4) and the homogeneous counterpart to (B1) 

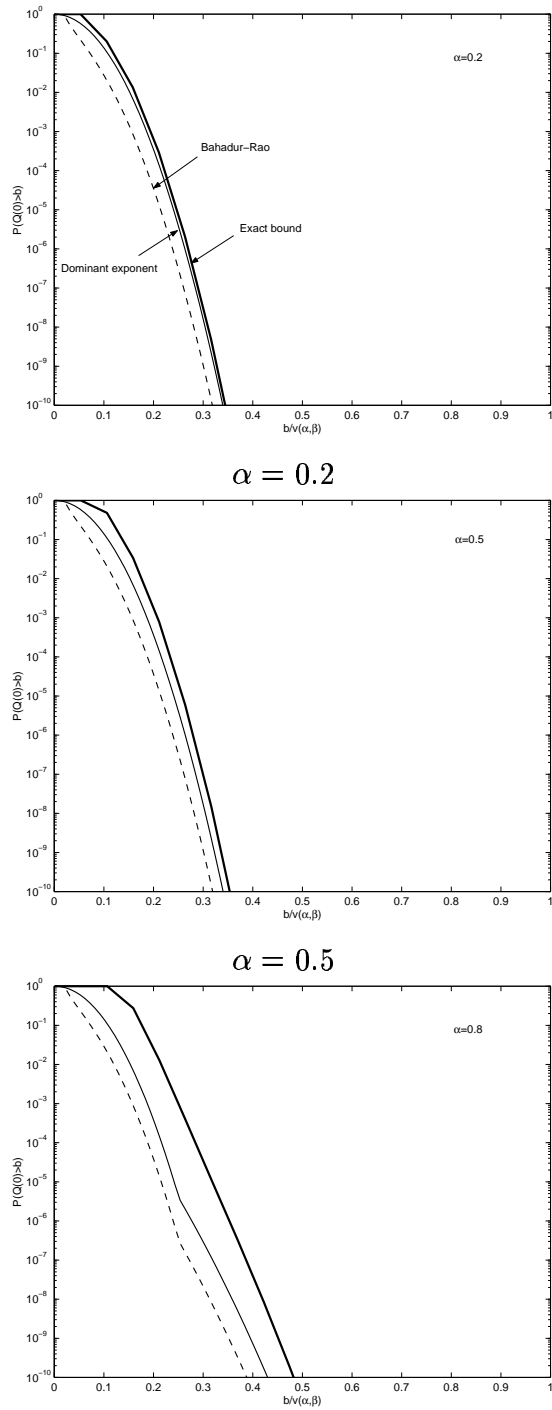

$\alpha=0.8$

Fig. 4. Exact (thick solid line), dominant probability (thin solid line), and Bahadur-Rao (dashed line) bounds to the backlog; the homogeneous case with $\rho_{i}=\alpha c / I, \sigma_{i}=5 \mathrm{MTU}, I=$ $100, c=150 \mathrm{Mbps}, \mathrm{MTU}=1500$ Bytes, $e=\mathrm{MTU} / c$.

becomes smaller; the bounds get fairly close to each other, and even, for very high utilization, (a) in (4) outperforms the homogeneous counterpart to (B1). It is also noteworthy that, contrary to (B1), the bound derived upon the assumption that the input flows are homogeneously regulated is not insensitive with respect to $\vec{\rho}$ for low to moderate utilization.

In Fig. 4, we show our exact bound (a) in (4), its dominant probability, and the Bahadur-Rao improvement for the setup given in the caption of the figure. We observe that Bahadur-Rao improvement is moderate; it is about an order of magnitude uniformly over the buffer size.

We compare our bounds to the backlog with Better than Poisson bound [7] in Fig. 5. We fix the aggregate arrival
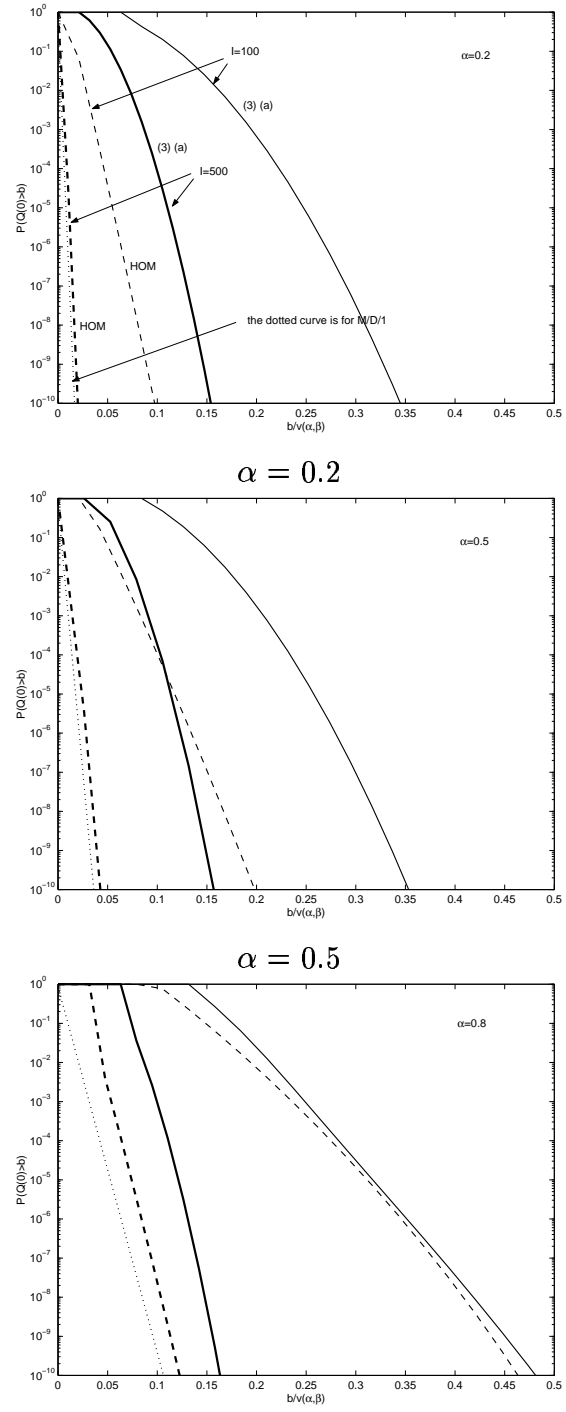

$\alpha=0.8$

Fig. 5. A comparison with Better than Poisson approach. The input flows are homogeneously regulated. We fix the aggregate arrival curve to $\alpha(t)=\rho t+\sigma$ with $\rho=\alpha c$ and $\sigma=500$ MTU; $c=150$ Mbps, $e=0$, MTU $=1500$ Bytes. The thick lines are for $I=500$; the thin lines are for $I=100$. Our bound (a) in (3) is shown as solid line; its homogeneous counterpart (Theorem 3 in [9]) as dashed line; and the asymptotic expansion for M/D/1 [7] as dotted line.

curve to $\alpha(t)=\rho t+\sigma$ with $\rho=\alpha c$ and $\sigma=500$ MTU. We show the results for three different utilizations $\alpha=0.2,0.5,0.8$, and for the number of input flows $I=100,500$. See the caption of Fig. 5 for the setup of other parameters. As pointed out in Section 3, our bounds admit the economy of scale, thus as we scale the buffer size and the service rate as $O(I)$, the bound on the back$\log$ decays exponentially with $I$. Equivalently, we fix the buffer size, service rate, and $\alpha$, but scale $\alpha_{i}$ as $O(1 / I)$. On the other hand, the Better than Poisson bound is invari- 
ant with respect to the number of input flows; it depends solely on $\rho$. The results in Fig. 5 confirm that our bounds decay with $I$. We observe that for $I=500$, which corresponds to $\sigma_{j}=1 \mathrm{MTU}$, our bound derived upon assuming the inputs are homogeneously regulated (Theorem 3 [9]), is fairly close to the Better than Poisson bound. For $I=100$, which corresponds to $\sigma_{j}=5 \mathrm{MTU}$, our bounds give a more pessimistic estimate than the Better than Poisson. This leads us to conclude that with bursty input flows the Better than Poisson approach may give over-optimistic estimate of the performance.

\section{Conclusion}

We propose a framework to derive probabilistic guarantees for networks with aggregate scheduling, e.g. expedited forwarding. Our approach is based on assumption that a node can be abstracted with a service curve, which is verified with the current definition of EF [1], [2]. A remarkable feature of our approach is that the bounds we obtain are exact, they are valid for any setting of the parameters, and in particular for any number of the inputs.

\section{ACKNOWLEDGMENT}

The authors thank Thomas Bonald and Alexandre Proutière for a discussion on [7]. A discussion with Don Towsley is also kindly acknowledged.

\section{REFERENCES}

[1] A. Charny, F. Baker, J. Bennett, K. Benson, J.-Y. Le Boudec, A. Chiu, W. Courtney, B. Davie, S. Davari, V. Firoiu, C. Kalmanek, K. K. Ramakrishnan, and D. Stiliadis, "Internetdraft: Ef phb redefined," November 2000.

[2] B. Davie (Editor), A. Charny, F. Baker, J. Bennett, K. Benson, J.-Y. Le Boudec, A. Chiu, W. Courtney, S. Davari, V. Firoiu, C. Kalmanek, K. K. Ramakrishnan, and D. Stiliadis, "Internetdraft: An expedited forwarding phb," April 2001.

[3] R. Agrawal, R. L. Cruz, C. Okino, and R. Rajan, "Performance bounds for flow control protocols," IEEE/ACM Transactions on Networking (7) 3, pp. 310-323, June 1999.

[4] C.S. Chang, "On deterministic traffic regulation and service guarantee: A systematic approach by filtering," IEEE Transactions on Information Theory, vol. 44, pp. 1096-1107, August 1998.

[5] J.-Y. Le Boudec, "Application of network calculus to guaranteed service networks," IEEE Transactions on Information Theory, vol. 44, pp. 1087-1096, May 1998.

[6] Jon C. R. Bennett, Kent Benson, Anna Charny, William F. Courtney, and Jean-Yves Le Boudec, "Delay jitter bounds and packet scale rate guarantee for expedited forwarding," in Proc. of IEEE INFOCOM'2001, March 2001.

[7] T. Bonald, A. Proutiére, and J. Roberts, "Statistical performance guarantees for streaming flows using expedited forwarding," in Proc. of IEEE INFOCOM'2001, March 2001.

[8] Cheng-Shang Chang, Wheyming Song, and Yuh ming Chiu, "On the performance of multiplexing independent regulated inputs," in Proc. of Sigmetrics 2001, Massachusetts, USA, May 2001.
[9] Milan Vojnović and Jean-Yves Le Boudec, "Bounds for independent regulated inputs multiplexed in a service curve network element," Tech. Rep. DSC/2001/015, Communication Systems Department, Swiss Federal Institute of Technology at Lausanne (EPFL), Lausanne, Switzerland, March 2001.

[10] J.-Y. Le Boudec and P. Thiran, Network Calculus, SpringerVerlag, 2001.

[11] Chen-Ming Chuang and Jin-Fu Chang, "Deterministic loss ratio quality of service guarantees for high speed networks," IEEE Communication Letters, vol. 4, no. 7, pp. 236-238, July 2000.

[12] Anna Charny and Jean-Yves Le Boudec, "Delay bounds in a network with aggregate scheduling," in First International Workshop on Quality of future Internet Services, September 2000.

[13] Wassily Hoeffding, "Probability inequalities for sums of bounded random variables," American Statistical Association Journal, pp. 13-30, March 1963.

[14] George Kesidis and Takis Konstantopoulos, "Worst-case performance of a buffer with independent shaped arrival processes," IEEE Communication Letters, 2000.

[15] D. Botvich and N. Duffield, "Large deviations, the shape of the loss curve, and economies of scale in large multiplexers," Queueing Systems, vol. 20, pp. 293-320, 1995.

[16] T. Konstantopoulos and G. Last, "On the dynamics and performance of stochastic fluid systems," Journal of Applied Probability, vol. 37, pp. 652-667, 2000.

[17] Nikolay Likhanov and Ravi R. Mazumdar, "Cell loss asymptotics in buffers fed with a large number of independent stationary sources," Journal of Applied Probability, vol. 36, pp. 86-96, 1999.

[18] R. R. Bahadur and R. Ranga Rao, "On deviations of the sample mean," Ann. Math. Statist., vol. 31, pp. 1015-1027, 1960.

[19] J. W. Roberts (Editor), COST 224: Performance evaluation and design of multiservice networks, Commision of the European Communities, 1991.

[20] M. Montgomery and G. de Veciana, "On the relevance of time scales in performance oriented traffic characterization," in Infocom'96, 1996, pp. 4d.3.1-4.d.3.8.

[21] Jean-Yves Le Boudec and Anna Charny, "Packet scale rate guarantee for non-fifo nodes," Tech. Rep. DSC200138, EPFL-DSC, http://dscwww.epfl.ch/EN/publications/documents/tr01_038.pdf, July 2001.

[22] Francois Baccelli and Pierre Brémaud, Elements of Queueing Theory, vol. 26, Applications of Mathematics, Springer-Verlag, 1991.

\section{APPENDIX}

\section{PROOF OF THEOREM 1}

From Lemma 2 [9], it holds

$\mathbb{P}(Q(t)>b) \leq \sum_{k=0}^{K-1} \mathbb{P}\left(A(t)-A\left(t-s_{k+1}\right)>b+\beta\left(s_{k}\right)\right)$,

for any $K \in \mathbb{N}$ and any $0=s_{0} \leq s_{1} \leq \cdots \leq s_{K}=\tau$.

Next, by Hoeffding's inequality for non-uniformly bounded random variables $0 \leq A_{i}(t)-A_{i}(s) \leq \alpha_{i}(t-s)$, for any $s \leq t$ (A3), we obtain $\mathbb{P}\left(A(t)-A\left(t-s_{k+1}\right)>\right.$ $\left.b+\beta\left(s_{k}\right)\right) \leq$

$$
\leq \exp \left(-\frac{2\left(\left(b+\beta\left(s_{k}\right)-\rho s_{k+1}\right)^{+}\right)^{2}}{\sum_{i=1}^{I} \alpha_{i}\left(s_{k+1}\right)^{2}}\right),
$$


where in the nominator of the exponent we use (A4).

On the other hand, in [9] (Inequality (14)) we show $\mathbb{P}\left(A(t)-A\left(t-s_{k+1}\right)>b+\beta\left(s_{k}\right)\right) \leq$

$$
\leq \exp \left(-\frac{\left(\left(b+\beta\left(s_{k}\right)-\rho s_{k+1}\right)^{+}\right)^{2}}{2 \sum_{i=1}^{I} v\left(\alpha_{i}, \lambda_{\rho_{i}}\right)^{2}}\right) .
$$

Finally, indeed, the minimum of (19) and (20) is an upper bound on $\mathbb{P}\left(A(t)-A\left(t-s_{k+1}\right)>b+\beta\left(s_{k}\right)\right)$. Using this minimum in (18) completes the proof.

\section{Proof of Theorem 2}

The first inequality in (4) is a corollary of Theorem 1 for leaky-bucket regulated inputs. The second inequality is obtained by upper-bounding the first term in the minimum operation in (4) (a) as follows

$$
\begin{aligned}
& \sum_{i=1}^{I}\left(\rho_{i} s_{k+1}+\sigma_{i}\right)^{2}= \\
& =\sum_{i=1}^{I} \rho_{i}^{2} s_{k+1}^{2}+2 \sum_{i=1}^{I} \rho_{i} \sigma_{i} s_{k+1}+\sum_{i=1}^{I} \sigma_{i}^{2} \\
& \leq \sum_{i=1}^{I} \rho_{i}^{2} s_{k+1}^{2}+2 \sqrt{\sum_{i=1}^{I} \rho_{i}^{2}} \sqrt{\sum_{i=1}^{I} \sigma_{i}^{2}} s_{k+1}+\sum_{i=1}^{I} \sigma_{i}^{2} \\
& =\left(\sqrt{\sum_{i=1}^{I} \rho_{i}^{2}} s_{k+1}+\sqrt{\sum_{i=1}^{I} \sigma_{i}^{2}}\right)^{2},
\end{aligned}
$$

where the inequation is by Cauchy-Schwartz's inequality.

The last inequality in (4) is by a trivial bound $\sum_{i=1}^{I} \rho_{i}^{2} \leq \rho^{2}$. This completes the proof of the theorem.

\section{PRoOF OF THEOREM 3}

Let $\tilde{A}^{*}=A \otimes \beta$. $A \otimes \beta$ is called the min-plus convolution of $A$ and $\beta$, defined by $(A \otimes \beta)(t)=\inf _{u \in[0, t]}\{A(t-$ $u)+\beta(u)\}$. By [8], [9], the infimum is obtained for $u \in[0, \tau]$, thus the majorizing process $\tilde{Q}(t)$ defined in Equation (2) satisfies $\tilde{Q}(t)=A(t)-\tilde{A}^{*}(t)$.

We now state and prove a preparatory lemma, and then continue with the proof of the theorem.

Lemma 1: We have

$$
\tilde{A}^{*}(t+u)-\tilde{A}^{*}(t) \leq u \hat{\beta} .
$$

Proof: Define $\gamma(u)=u \hat{\beta} 1\{u \geq 0\}$. It follows from the definition of $\hat{\beta}$ that, for all $0 \leq s \leq t$ :

$$
\beta(t-s)+\gamma(s) \geq \beta(t),
$$

thus

$$
\beta \otimes \gamma \geq \beta
$$

It follows that

$$
\tilde{A}^{*}=A \otimes \beta \leq A \otimes(\beta \otimes \gamma)=(A \otimes \beta) \otimes \gamma=\tilde{A}^{*} \otimes \gamma .
$$

Coming back to the definition of $\otimes$ we find that

$$
\tilde{A}^{*}(t+u) \leq \tilde{A}^{*}(t)+u \hat{\beta} .
$$

For a wide-sense increasing measurable function $\varphi$ such that $\varphi^{\prime}=\psi$

$$
\varphi(\tilde{Q}(t))-\varphi(\tilde{Q}(0))=\int_{0}^{t} \varphi^{\prime}(\tilde{Q}(s)) \tilde{Q}(d s),
$$

where $\tilde{Q}(d s)=A(d s)-\tilde{A}^{*}(d s)$.

It follows from the lemma that

$$
\int_{0}^{t} \psi(\tilde{Q}(s)) \tilde{A}^{*}(d s) \leq \hat{\beta} \int_{0}^{t} \psi(\tilde{Q}(s)) d s .
$$

Combining (21) with (22) we obtain

$\varphi(\tilde{Q}(t))-\varphi(\tilde{Q}(0)) \leq \int_{0}^{t} \psi(\tilde{Q}(s)) A(d s)-\hat{\beta} \int_{0}^{t} \psi(\tilde{Q}(s)) d s$.

Take the expectation at both sides to obtain

$$
0 \leq \bar{\rho} t \mathbb{E}_{A}\left[\varphi^{\prime}(\tilde{Q}(0))\right]-\hat{\beta} t \mathbb{E}\left[\varphi^{\prime}(\tilde{Q}(0))\right],
$$

where the Palm expectation is by Campbell's formula [22]. Replacing $\varphi^{\prime}$ with $\psi$ we prove (5).

\section{Proof of Theorem 4}

By the service curve property, there exists $s \leq t$ such that $A^{*}(t) \geq A^{\prime}(s)+\beta(t-s)$, where $A^{\prime}(s)=A(s)-L(s)$. Note $L(t)-L(t-1)=(L(t)-L(t-1)) 1_{Q(t)=B}$. For $s=t, A^{\prime}(s)+\beta(t-s) \geq A^{\prime}(t)$, and thus $Q(t)=0$. Since we are interested in the events $\{Q(t)=B\}$, we are allowed to only consider $s<t$. Then

$$
\begin{aligned}
& L(t)-L(t-1)= \\
& =A(t)-A^{*}(t)-Q(t)-L(t-1) \\
& \leq A(t)-A(s)-\beta(t-s)-Q(t)+L(s)-L(t-1) \\
& \leq A(t)-A(s)-\beta(t-s)-Q(t) .
\end{aligned}
$$

And,

$$
\begin{aligned}
& (L(t)-L(t-1)) 1_{Q(t)=B} \leq \\
& \leq(A(t)-A(s)-\beta(t-s)-Q(t)) 1_{Q(t)=B} \\
& =(A(t)-A(s)-\beta(t-s)-B) 1_{Q(t)=B} \\
& \leq(A(t)-A(s)-\beta(t-s)-B) \vee 0 \\
& \leq\left(\max _{0 \leq s \leq t}\{A(t)-A(s)-\beta(t-s)\}-B\right) \vee 0 \\
& =\left(Q^{\infty}(t)-B\right) \vee 0 .
\end{aligned}
$$

Thus, $\mathbb{P}(L(t)-L(t-1)>u) \leq \mathbb{P}\left(Q^{\infty}(t) \vee B>u+B\right)=$ $\mathbb{P}\left(Q^{\infty}(t)>u+B\right)$, for $u \geq 0$, and then

$$
\mathbb{E}[L(t)-L(t-1)] \leq \int_{0}^{\hat{l}(1) \alpha(1)} \mathbb{P}\left(Q^{\infty}(t)>u+B\right) d u,
$$

where the upper boundary in the integral is the deterministic upper bound on the lost data $L(s)-L(0) \leq A(s) \hat{l}(s) \leq$ $\alpha(s) \hat{l}(s)$, for any $s \geq 0 ; \hat{l}(\cdot)$ is the generalized Chuang and Chang's bound [11], [10].

The bound to the loss ratio (7) is immediately obtained by observing $\bar{l}=\mathbb{E}[L(t)-L(t-1)] / \mathbb{E}[A(t)-A(t-1)]$, and by definition $\bar{\rho}=\mathbb{E}[A(t)-A(t-1)]$. This completes the proof of the theorem. 